appeared to have an overall beneficial effect on alcohol consumption.

Conclusions: Further research is needed to evaluate the specific impacts on regular amphetamine use and to develop more efficacious interventions among regular cannabis users. A stepped-care approach to interventions for excessive alcohol consumption among people with a psychotic disorder is recommended.

01-07

\section{Cannabis use as a complication in psychological treatments for people at ultrahigh risk of psychosis: early findings from the DEPTh trial}

\section{Startup ${ }^{1,2}$, V Carr ${ }^{2,3}$, A Baker ${ }^{2,3}$, H Stain ${ }^{3,4}$, S Halpin', S Bucci ${ }^{5}$}

${ }^{1}$ School of Psychology, The University of Newcastle, Australia; ${ }^{2}$ Centre for Mental Health Studies, The University of Newcastle; ${ }^{3}$ Neuroscience Institute of Schizophrenia and Allied Disorders (NISAD), New South Wales, Australia; ${ }^{4}$ Centre for Rural \& Remote Mental Health, The University of Newcastle; ${ }^{5}$ sychological

Assistance Service, Hunter New England Area Health Service, Newcastle, Australia

The DEPTh project is a randomized controlled trial comparing the effectiveness of cognitive behaviour therapy (CBT) and person-centered therapy for the prevention of psychosis among young people who are at ultrahigh risk. It is being conducted on two sites in New South Wales: the Hunter Valley and the Greater Western Area. The CBT is modeled on the manual developed by Paul French and Tony Morrison in the UK. This form of CBT has been shown in one trial to be effective in preventing transition to psychosis. However, it does not include any techniques that specifically focus on the reduction of substance abuse even though such abuse, especially of cannabis, is known to be very high among people who are at ultrahigh risk, and cannabis use has been found in prospective studies to be associated with the later onset of psychosis. Therefore, we have adapted French and Morrison's CBT by integrating it with a manual for motivational interviewing (MI) with young cannabis users. MI is directed toward the resolution of ambivalence and movement toward change, with the resources and motivation to change considered to reside within the client. The therapist's task is to create a set of conditions that will enhance the client's own motivation and commitment for change by drawing on their personal perceptions, goals and values. The ways in which CBT and MI are integrated in the DEPTh project will be illustrated with case material.

\section{Personality Disorders (International Society for the Study of Personality Disorders Symposium)}

\author{
AM Chanen \\ ORYGEN Research Centre, Parkville, Victoria, Australia
}

\section{Overview}

This symposium brings together researchers from Australia and New Zealand affiliated with the International Society for the Study of Personality Disorders. It canvasses current personality disorder research from neurobiology through to interpersonal processes and intervention across the life span from youth to old age.

02-01

\section{Emotion processing and regulation in first-presentation borderline personality disorder}

\section{NB Allen, S Chong, AM Chanen}

ORYGEN Research Centre and Department of Psychology, The University of Melbourne, Melbourne, Australia

Emotion dysregulation is widely believed to be one of the core features of borderline personality disorder (BPD). To date, there has been no study examining psychophysiological and self-report measures of deliberate regulation of emotions in BPD in those in the early phases of the disorder. The aims of the study were to measure the 1) psychophysiological and subjective emotional response and 2) psychophysiological and subjective ability to regulate one's emotional response to emotional images in 15- to 24-year-olds with first-presentation BPD compared with healthy controls. Twenty patients with full-syndrome BPD and 20 healthy comparison participants were examined. Test stimuli used were a set of standardized photographic images with pleasant, neutral or unpleasant valence. Participants were also instructed to either 'maintain' or 'suppress' the emotional response they were having to the stimuli. In addition to self-report ratings, emotional responses to the stimuli were measured by startle response and skin conductance. Contrary to the hypotheses, self-report and psychophysiological data did not provide evidence that the BPD participants reacted with more intense affective responses 